\title{
Solidification Chronology of the Metal Matrix and a Study of Conditions for Micropore Formation in Cast Irons Using EPMA and FTA
}

\author{
Björn Domeij ${ }^{1, a^{*}}$, Attila Diószegi ${ }^{1, b}$ \\ 1Jönköping University, Gjuterigatan 5, SE-551 11 Jönköping, Sweden \\ äBjorn.Domeij@ju.se, ${ }^{b}$ Attila.Dioszegi@ju.se
}

Keywords: Microsegregation, Microporosity, Cast Iron, Solidification, EPMA

\begin{abstract}
Microsegregation is closely coupled with solidification, the development of microstructure, and involved in the formation of various casting defects. This paper demonstrates how the local composition of the metal matrix of graphitic cast irons, measured using quantitative electron microprobe analysis, can be used to estimate its solidification chronology. The method is applied in combination with Fourier thermal analysis to investigate the formation of micropores in cast irons with varying proportions of compacted and spheroidal graphite produced by remelting. The results indicate that micropores formed at mass fractions of solid between 0.77 and 0.91 , which corresponded to a stage of solidification when the temperature decline of the castings was large and increasing. In 4 out of the 5 castings, pores appear to have formed soon after the rate of solidification and heat dissipation had reached their maximum and were decreasing. Freezing point depression due to build-up of microsegregation and the transition from compacted to spheroidal type eutectic growth both influence solidification kinetics and the temperature evolution of the casting, but did not clearly account for the observed late decline in the rate of solidification.
\end{abstract}

\section{Introduction}

Micropores is a class of pores, characterized by their complex shape, owing to formation at a late stage of solidification when solid microstructure already well developed. These defects can be costly, as they typically remain undetected until uncovered during machining operations [1], or worse, cause product malfunction during usage, e.g. leakage of fluids and gases through the component. A large number of material and process variables contribute to the complex conditions of the defect's formation [2]. It is commonly accepted that the formation of micropores in cast irons relates to volume changes which occur during the later stages of solidification [3]. However, since the rupture of liquid metal requires tensions exceeding the yield strength of the surrounding solid, it is commonly argued that pores likely nucleate at an earlier stage of solidification but grow to a substantial size during the later stage [4]. By formation of micropores, this paper therefore refers to the latter process during which the predominant shape and size of the pore found in the final casting develops. In long freezing range alloys, micropores may form by feeding of atmospheric gases from the surface into the casting [4]. This type of micropore has for example been observed in hypoeutectic lamellar graphite irons, where pores were connected to the surface of the casting in a region where a hotspot was close to the mold wall [6]. In this case, pores were observed to occupy the space between dendrite arms and eutectic cells, which was attributed to an increased permeability of the columnar zone, related to the hotspot [7]. A later study also indicates that pores are typically located on or close to grain boundaries [8]. Short freezing range alloys more often feature isolated micropores [4]. This should be more typical for compacted and spheroidal graphite irons due to their near eutectic composition. It is also well known that for a given graphite content, SGI typically demands more feeding than LGI and CGI [9]. This has been proposed to relate to the growth of graphite by plastic deformation of the surrounding austenite, which causes some of the graphite expansion to be translated onto the solid structure, leading to an increase in the volume of the casting instead of feeding [5]. While it is commonly accepted that micropores (i.e. their final size and shape) develop at a late stage of solidification [3], this paper attempts to estimate more 
precisely the stage of solidification and conditions in the casting associated with formation of micropores.

\section{Material}

The investigated material was initially cast as a SGI which was later resolidified in a laboratory furnace using varying holding time at maximum temperature. The chemical composition in Table 1 was measured on a coin sample produced just before pouring, using a spark optical emission spectrometer (OES). FeSi inoculant was introduced to the stream during pouring. In preparation for the resolidification experiments, cylindrical rods with a diameter of $\varnothing 38 \mathrm{~mm}$ were machined from the base material. The length of the rods was adjusted such that the weight was $400 \pm 0.5 \mathrm{~g}$.

Table 1. Material chemical composition given in weight percent, measured using OES. Carbon equivalent $\mathrm{CE}=\mathrm{C}+(\mathrm{Si}+\mathrm{P}) / 3$.

\begin{tabular}{lllllllll}
\hline $\mathrm{CE}$ & $\mathrm{C}$ & $\mathrm{Si}$ & $\mathrm{Mn}$ & $\mathrm{S}$ & $\mathrm{P}$ & $\mathrm{Cu}$ & $\mathrm{Sn}$ & $\mathrm{Mg}$ \\
\hline 4.72 & 3.86 & 2.59 & 0.64 & 0.01 & 0.03 & 0.84 & 0.098 & 0.065
\end{tabular}

\section{Experiment}

To produce cast irons of varying graphite morphology, the base material was remelted in a laboratory furnace. The experimental setup is essentially as described in a previous publication [10] with few modifications. Ar (99.999 vol\% purity) is introduced to the furnace through a hole in the bottom lid at a rate of $5 \mathrm{l} / \mathrm{min}$. Excess gas is vented through a hole in the top lid. The machined specimen of the base material was placed in a cylindrical alumina crucible of inner dimensions Ø40x60 mm and placed in the middle of the furnace, resting on a column of graphite cylinders. Three S-type thermocouples protected by quartz glass tubes were lowered into the furnace through holes in the lid, such that their welds were located $20 \mathrm{~mm}$ above the floor of the crucible. The three thermocouples, TC1, TC2 and TC3 were placed in the center of the specimen, against the inner wall, and outside the crucible respectively. The position in relation to the lid was marked, after which they were raised to an offset of $200 \mathrm{~mm}$ to avoid unnecessary exposure to high temperatures while not recording. The furnace was programmed to ramp up to $1450{ }^{\circ} \mathrm{C}$ over $75 \mathrm{~min}$, and then dwell for a duration according to Table 2, after which it was turned off. The thermocouples were lowered to the predetermined position 5 to $10 \mathrm{~min}$ prior to the end of the dwelling time after which recording was started. More details about the experimental method are published elsewhere [11].

Table 2. List of the specimens, furnace holding time, and the resulting measured nodularity (ISO 16112).

\begin{tabular}{llllll} 
Alias & H10 & H20 & H30 & H40 & H50 \\
\hline Dwell time & $10 \mathrm{~min}$ & $20 \mathrm{~min}$ & $30 \mathrm{~min}$ & $40 \mathrm{~min}$ & $50 \mathrm{~min}$ \\
Nodularity & $87 \%$ & $69 \%$ & $54 \%$ & $38 \%$ & $22 \%$ \\
\hline
\end{tabular}

\section{Investigation Methods}

Sample preparation and graphite analysis. The specimens were cut along the plane $20 \mathrm{~mm}$ above the bottom of the specimen, producing a circular cross section. The bottom half was mounted in a $\varnothing 50 \mathrm{~mm}$ disc of Bakelite with carbon filler. The cross sections were ground on silica paper down to a grit size of P2000 (FEPA) and polished using $3 \mu \mathrm{m}$ diamond suspension on a satin woven acetate cloth. A final polishing step was performed using an oxide slurry on a porous neoprene cloth. The percent nodularity presented in Table 2 was measured according to ISO 16112 using an optical microscope at $5 \mathrm{x}$ objective lens magnification and digital image analysis on a sample area of at least $151 \mathrm{~mm}^{2}$ for each specimen. The surface was then color etched [12] to obtain an overview of the microsegregation patterns of Si. Prior to EPMA the last polishing step was repeated and the mounting was removed. 
EPMA-WDS. In each specimen, a $3 \times 3 \mathrm{~mm}$ sound area and a $1 \times 1 \mathrm{~mm}$ porous area of the cross section were mapped along a 300×300 and a 200x200 point raster respectively, using quantitative EPMA. The microprobe is a modified ARL-SEMQ equipped with six WDS spectrometers. The probe uses an acceleration voltage of $25 \mathrm{kV}$ and a sample current of $6 \mu \mathrm{A}$. Each measurement is an average over a circle with a diameter corresponding to the spacing of the raster and a dwell time of 0.2 s. The spectrometers were set up to measure $\mathrm{C}, \mathrm{Si}, \mathrm{Mn}, \mathrm{P}, \mathrm{Cu}$, and $\mathrm{Sn}$. A crystal suitable for light elements was used in the spectrometer set up to measure C. Calibration was performed using certified reference materials, covering the composition range of the investigated material.

Microsegregation and the solidification chronology of the metal matrix. During solidification, alloying elements partition between the liquid and the solid. Since diffusion of solute is relatively slow in the solid, there is typically not enough time for solute to equilibrate within it, meaning equilibrium only holds true locally, over the solid-liquid interface. The composition of the liquid therefore evolves as solidification proceeds, leaving composition profiles in the solid. For elements which diffuse slowly, these profiles may be well preserved in the final cast component. The profiles can be plotted as the solute concentration in the solid as a function of mass fraction of solid. From here on, this is what is meant by "solute profile". Solute profiles may serve as maps to associate the local composition in any point of the metal matrix with a mass fraction solid, and thereby determine the solidification chronology of the metal matrix. Applied to a quantitative elemental map with sufficient resolution, this approach allows for estimation of the evolution of the microstructure as a function of mass fraction solid. In this paper, solute profiles are both constructed from EPMA measurements and theoretically calculated as explained below.

(a) solute concentration as a function of solid fraction can be measured in binary alloys using a combination of electron probe microanalysis (EPMA) and a traditional point counting method [13]. For the present multi-component alloy, the raster points are assumed to have solidified according to descending order of $T_{E}^{*}$ according to Eq. (1), where $\Delta w_{i}$ is the deviation from the average weight fraction of element $i$. $T_{E}^{*}$ is an estimation of equilibrium eutectic temperature $T_{E}$ found by fitting a hyperplane of $\Delta w_{i}$ to $T_{E}$ calculated using Thermo-Calc (TCFE7) for the composition range of the alloy. The justification for this approach is that each element contributes to a lowering of the equilibrium eutectic temperature $T_{E}$ in the melt [14], meaning a lower value of $T_{E}$ corresponds to a solidification at a lower temperature. Raster points including graphite, defined as points where $\mathrm{C}$ exceeds 7 wt.\%, were excluded from the analysis since Graphite contains almost exclusively carbon. The analysis therefore concerns only the partitioning between the metal matrix and the liquid.

$$
T_{E}^{*} \approx 1158.86+3.77 * \Delta w_{S i}-3.43 * \Delta w_{M n}-18.93 * \Delta w_{P}+3.30 * \Delta w_{C u}
$$

(b) the solute profile can be estimated using the Gulliver-Scheil solidification simulation module of Thermo-Calc which steps down in temperature, updating phase equilibriums under the assumptions of Gullive-Scheil segregation of solute.

(c) the solute profile can be estimated from the initial composition and the partition coefficient using the Gulliver-Scheil equation.

Assuming the density of the metal matrix is approximately uniform, the point fraction obtained using (a) corresponds approximately to a mass fraction. Moreover, the evolution of the mass fraction graphite is unknown for (a) and (c). In order to compare the solute profiles, the mass fractions of the metal matrix are normalized by the final mass fractions. Since graphite constitutes a small mass fraction of the matrix, and precipitates approximately in proportion to the austenite as part of the eutectic, this normalized mass fraction is approximately equal to the total mass fraction of solid. 
Solid fraction of pore formation. The introduced approach is applied to investigate the conditions in the casting during micropore formation. The first step is to find the mass fraction of solid corresponding to the formation of the pore, from here on referred to as the solid fraction of pore formation $\left(f_{S}^{\text {pore }}\right)$. To find $f_{S}^{\text {pore }}$, it is considered that solidification was interrupted locally by displacing liquid from some of the solid structure, and that the solid fraction translated from the composition near the surface of this structure using the previously described approach corresponds to the solid fraction when this occurred. The Mn profile was chosen for the translation because it is well above the detection limit throughout the metal matrix and is steadily increasing towards the last melt to solidify. The latter is beneficial because the measurement on the metal matrix closest to the pore can be defined as the maximum concentration Mn before the probe passes over the edge and the value drops. Concave segments of the pore typically feature higher concentration of Mn are considered likely to correspond to interfaces towards the liquid after pore formation. For each material specimen, the mean of the highest values of Mn near convex segments of the pore surface was sampled from elemental maps like Figure 1. These mean concentrations were then translated into solid fractions using solute profiles by linear interpolation for method (a) and (b) as illustrated in Figure 2, and by solving the Scheil equation for the solid fraction for method (c).

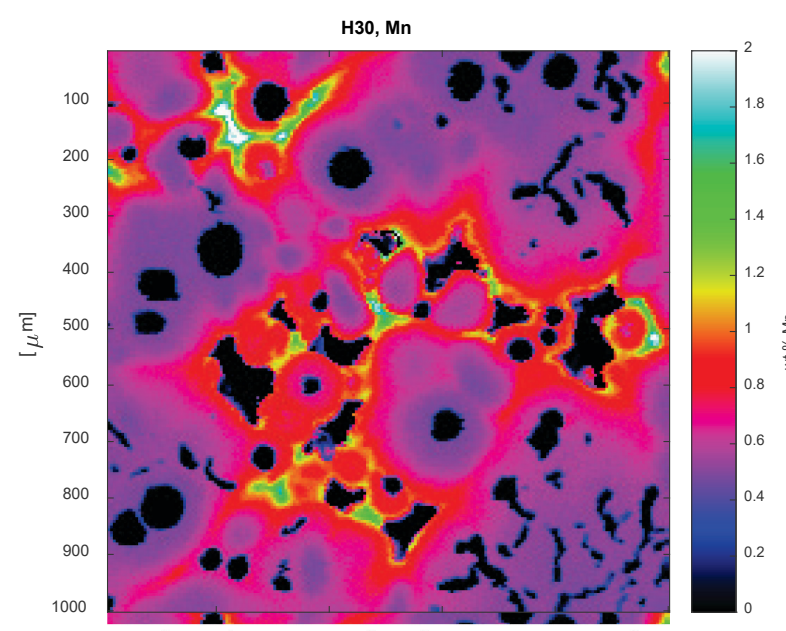

Figure 1. A quantitative map of the $\mathrm{Mn}$ concentration of a porous area of the cross section of material H30. The high concentration of $\mathrm{Mn}$ in the viscinity of pores helps distinguishing them from the graphite particles.

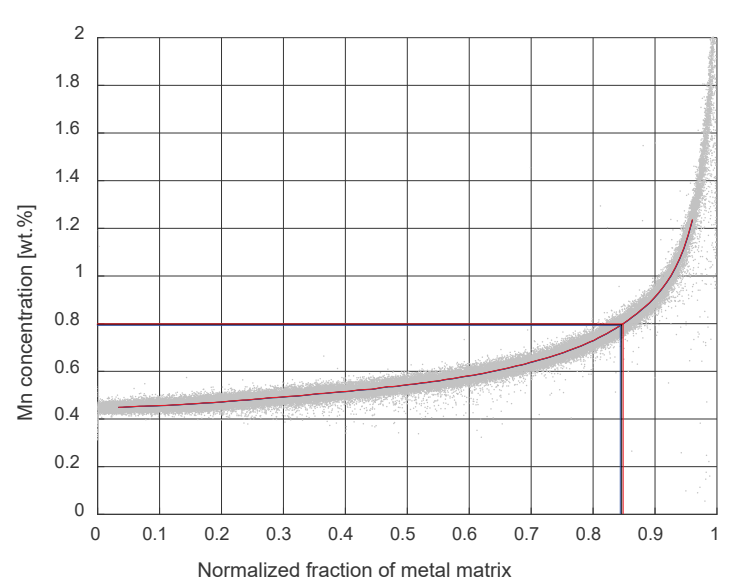

Figure 2. Grey dots represent EPMA measurements from a sound cross section of H30. The smooth profile was obtained using moving a moving average filter. Horizontal and vertical lines illustrate how local Mn concentrations were translated to solid fractions.

Connecting the solidification chronology to thermal analysis. The evolution of $f_{S}$ as a function of time may be estimated using Fourier Thermal Analysis (FTA) [15] for cooling curves from thermocouples TC1 and TC2. The solid fraction associated with the local composition in any point of the metal matrix corresponds to a point in time in the thermal analysis, and can thereby be linked the to FTA analysis. The FTA script in the referenced paper was developed for a hypoeutectic cast iron. To suit the present alloy, the script was adjusted to model solidification as purely eutectic, affecting mainly the evolution of $C_{V}$ since it is updated according to the instantaneous volume fractions of the component phases $C_{V}=f_{L} C_{V}^{L}+f_{\gamma} C_{V}^{\gamma}+f_{G} C_{V}^{G}$. Parameters in

Table 3 used in the analysis were calculated using the Poly 3 module of the ThermoCalc Software with the TCFE7 databank [16] for the composition in Table 1. Sn was excluded since it is not included in TFCE7. The validity of FTA for the present experimental conditions are essentially the same as discussed in an earlier paper [10]. 
Table 3. Input parameters for the Fourier Thermal Analysis.

\begin{tabular}{|c|l|c|l|}
\hline Symbol & Value & Unit & Description \\
\hline$\rho_{L}$ & 6741 & $\mathrm{~kg} / \mathrm{m}^{3}$ & Density of Liquid \\
\hline$\rho_{\gamma}$ & 7298 & $\mathrm{~kg} / \mathrm{m}^{3}$ & Density of Austenite \\
\hline$\rho_{G}$ & 2197 & $\mathrm{~kg} / \mathrm{m}^{3}$ & Density of Graphite \\
\hline$C_{P}^{L}$ & 827 & $\mathrm{~J} /(\mathrm{kgK})$ & Specific Heat Capacity of Liquid \\
\hline$C_{P}^{\gamma}$ & 873 & $\mathrm{~J} /(\mathrm{kgK})$ & Specific Heat Capacity of Austenite \\
\hline$C_{P}^{G}$ & 1968 & $\mathrm{~J} /(\mathrm{kgK})$ & Specific Heat Capacity of Graphite \\
\hline$f_{G}^{E}$ & 7.5 & $\%$ & Volume fraction Gr at end of solidification \\
\hline
\end{tabular}

\section{Results and Discussion}

Solid fraction of pore formation. The estimated $f_{S}^{\text {pore }}$ are presented in Figure 3. The values are similar for methods (a), (b) and (c), meaning reasonable accuracy can be achieved using a theoretical profile. However, since the degree of agreement between empirical and theoretical profiles depends on the considered solute element, the material and its solidification conditions, as well as the accuracy of the input data for the calculations, the use of an empirical profile is preferred. For this reason, further discussion concerns $f_{S}^{\text {pore }}$ determined using the empirical profiles.

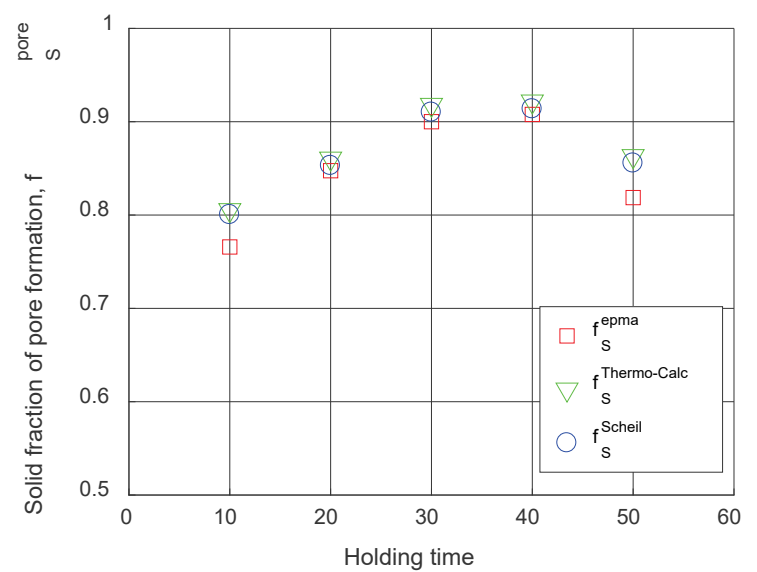

Figure 3. Mass fractions of solid at pore formation.

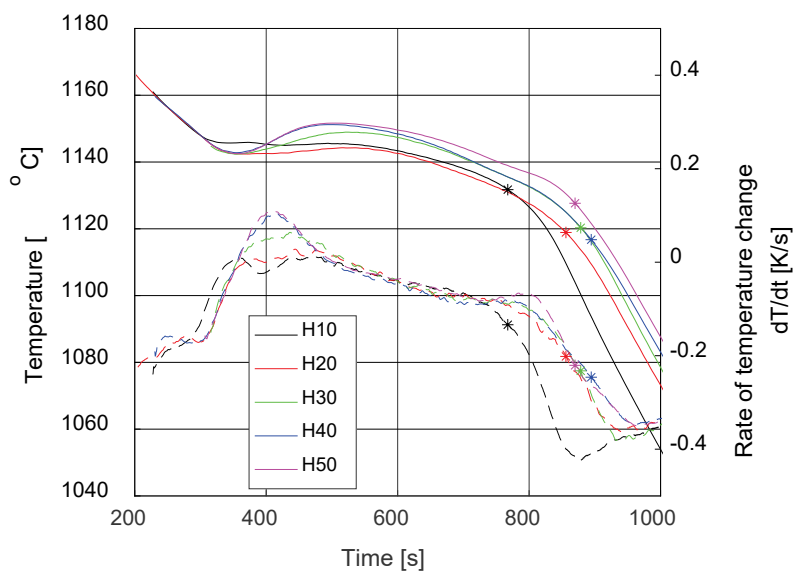

Figure 4. Cooling curves (-), their time derivatives (--), and the solid fraction of pore formation $(*)$.

The trend indicated in Figure 3 is not statistically significant as this investigation was limited to only one sample of each material. What can be said, however, is that pore formation occurred between a solid fraction of 0.77 and 0.91 .

Thermal conditions and solidification kinetics. Viewing Figure 4, we find that $f_{S}^{\text {pore }}$ is associated with a segment of the cooling curves where the decline in temperature is rapidly increasing. The temperature of the casting is determined by the balance between dissipated heat and internal generated heat which are associated with the time derivative of the zero line in Figure 5 and the solidification rate in Figure 6 respectively. These figures indicate that for $\mathrm{H} 20$ to H50, pores formed soon after the maximum rate of both heat dissipation and of solidification occurred and was on decline. A plausible mechanism for the formation of the micropores therefore appears to be a combination of a decreased rate of solidification and an increased temperature decline, contributing to an imbalance between the graphite expansion and thermal contraction of the phases. In combination with the large resistance to feeding at this stage of solidification, this could cause a build-up of tension in the liquid which is instead relieved by the formation of pores. 


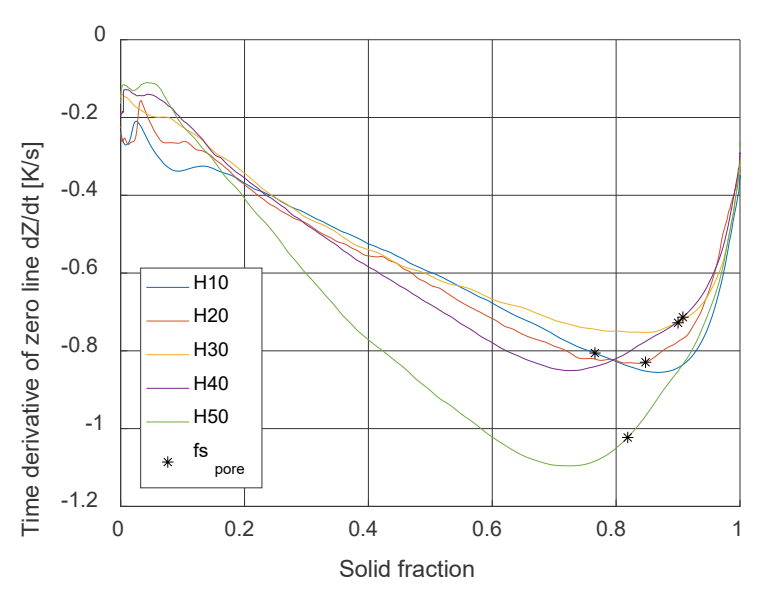

Figure 5. Time derivative of the zero line Asterix indicates the solid fraction of pore formation.

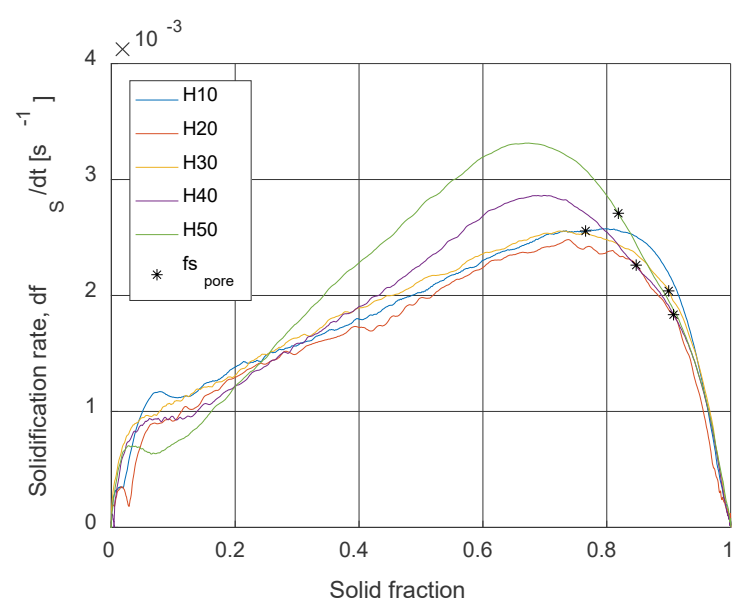

Figure 6. Solidification rate. Asterix indicates the solid fraction of pore formation.

Microsegregation and undercooling. Closely related to the temperature and the rate of solidification is the depression of the freezing point in the residual liquid due to microsegregation of solute. Here the freezing point is assumed to be the equilibrium eutectic temperature of the liquid $T_{E}^{L}$ and the eutectic undercooling of the liquid is defined as $\Delta T_{E}^{L}=T_{E}^{L}-T$. Since the driving force for the eutectic reaction is proportional to this value, the late drop in the rate of solidification observed in Figure 6 could be explained by a decrease in $\Delta T_{E}^{L} . T_{E}^{L}$ as a function of solid fraction was estimated using the Gulliver-Scheil solidification simulation module of Thermo-Calc after which $\Delta T_{E}^{L}$ was calculated by subtraction of the measured temperature. $\Delta T_{E}^{L}$ for TC1 and TC2 are plotted in Figure 7 and Figure 8 respectively.

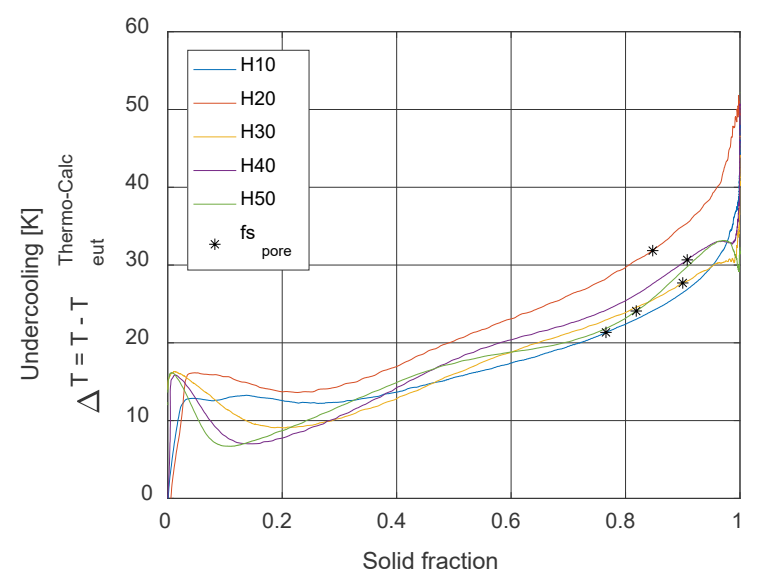

Figure 7. Estimated evolution of undercooling for TC1.

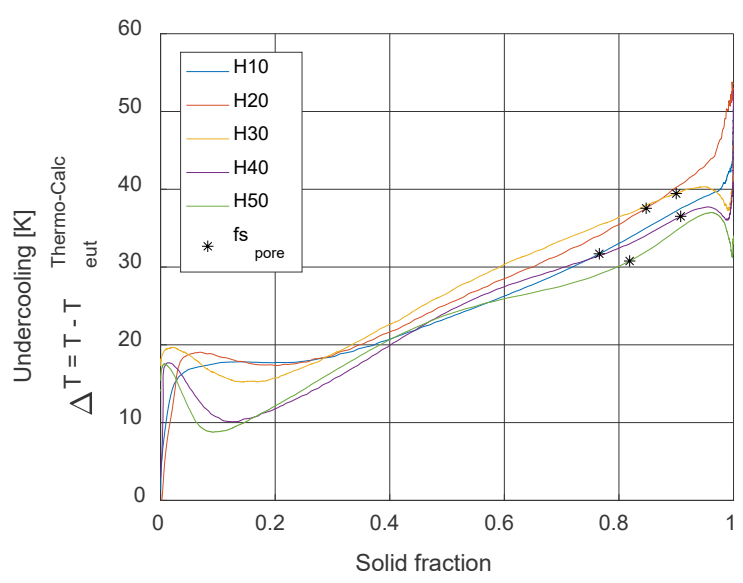

Figure 8. Estimated evolution of undercooling for TC2.

Figure 7 and Figure 8 indicates that the calculated undercooling in all cases increased through $f_{S}^{\text {pore }}$. It therefore appears that the rate of solidification decreased in spite of increasing $\Delta T_{E}^{L}$. For this reason it appears unlikely that the freezing point depression of $T_{E}^{L}$, caused by microsegregation of solute, played a decisive role in the late decrease in the rate of solidification observed in Figure 6. On a side note, Figure 7 and Figure 8 interestingly suggest that nodularity (see Table 2) mainly influence $\Delta T_{E}^{L}$ during the earlier half of solidification. The transient increase or decrease of $\Delta T_{E}^{L}$ that occurs as $f_{S} \rightarrow 1$ is associated with high uncertainty as this depends heavily on the temperature chosen as end of solidification in the thermal analysis. 
CG-SG transition. Another factor known to influence the rate of solidification is the growth mode of the graphite-austenite eutectic. The lamellar and compacted shape of the graphite grows in collaboration with austenite with both phases in contact with the liquid [17, 18]. Spheroidal graphite on the other hand grows predominantly at a slower rate, limited by diffusion of carbon through a surrounding layer of austenite [18]. For mixtures of spheroidal and compacted graphite, the relative amount and development of the two morphologies is therefore of importance. Using a similar approach as for construction of the solute profiles, the evolution of the microstructure of the metal matrix as a function of mass fraction of solid can be estimated based on the EPMA maps [19]. indicates that the austenite growing from the center of the compacted graphite cells eventually reaches and grows past the final extent of compacted graphite tips as shown in Figure 9. This is interpreted as the solid fraction by which the austenite envelopes the compacted graphite tips, after which growth proceeds in a manner similar to spheroidal graphite, by diffusion of carbon through a layer of austenite. The estimated start and end of this transition, here referred to as the CG-SG transition, is presented in Figure 10 in relation to the rate of solidification.

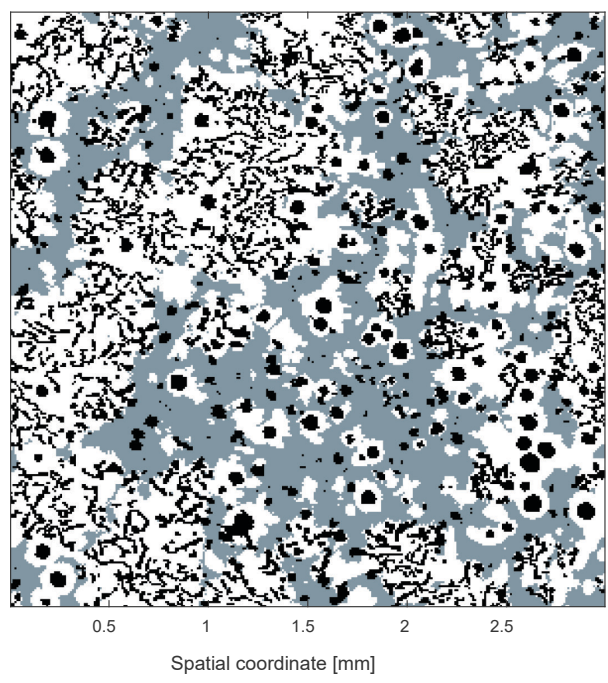

Figure 9. Reconstruction of microstructure based on the $3 \times 3$ EPMA for H50 at fs=0.6. White: solid, grey: liquid, black: graphite.

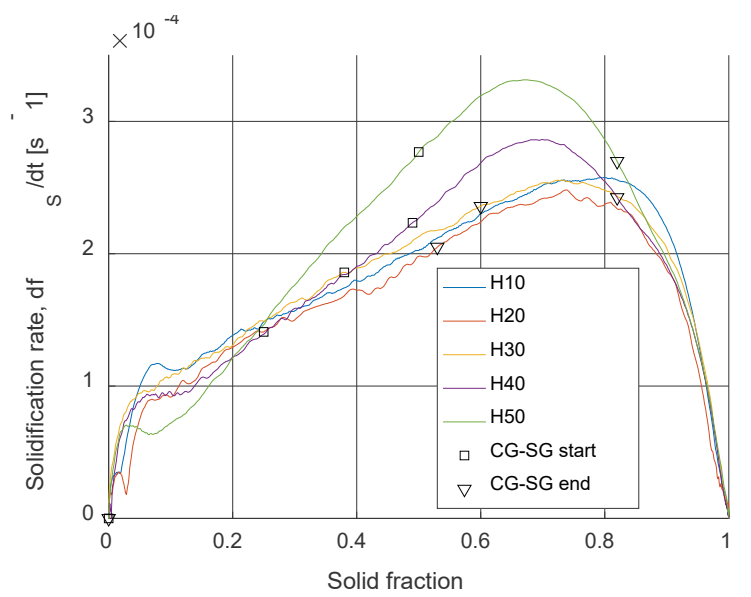

Figure 10. Solidification rate and the compacted to spheroidal transition. Squares and triangles indicate the first and last compacted graphite tip in the EPMA map to be overgrown by the metal matrix.

For high nodularities the transition occurs prior to the maximum rate of solidification. However, for $\mathrm{H} 40$ and $\mathrm{H} 50$, corresponding to $38 \%$ and $22 \%$ nodularity respectively, the maximum rate of solidification appears to occur during the transition. It is possible that this is coincidental, but it appears important to note that the maximum occurs at a lower solid fraction in these materials. Also, if the CG-SG transition has an impact on the rate of solidification, it would be surprising if the effect was not greater in materials with higher fraction compacted graphite.

Remarks. It is widely accepted that the interfacial surface area between solid and liquid drops during the later stage of solidification due to impingement of solidification units, which reduces the rate of solidification [20]. It is plausible that this is the major factor controlling the rate of solidification during the late stage of solidification, however the evolution of the solid-liquid interfacial surface area is a subject for future research.

\section{Conclusions}

This paper has demonstrated how the local chemical composition of the metal matrix of graphitic cast irons can be used to estimate its solidification chronology and how this may be connected to thermal analysis. This approach was applied to investigate the conditions for micropore formation in a small series of cast irons with varying nodularity. Solidification was locally interrupted by the 
formation of micropores at mass fractions of solid between 0.77 and 0.91 which corresponded to a time when the temperature decline of the casting was increasing. In 4 out of the 5 cases, pore formation appears to have occurred after the solidification rate and heat dissipation had reached their maximum and were on decline. The combination of the deceleration of solidification and the accelerated temperature decline appears to have been important for the formation of micropores in the studied materials. While both the decrease of the freezing point due to build-up of microsegregation and the transition from compacted to spheroidal type growth play a role in the kinetics and temperature evolution of the solidifying casting, neither appears to clearly account for the observed decrease in the rate of solidification which was associated with micropore formation.

\section{Acknowledgements}

This work was carried out as part of a research project "Spofic II" within the Swedish Casting Innovation Center (CIC), financed by VINNOVA-Swedish Governmental Agency for Innovation Systems (2013-04720). The project is a collaboration between Scania CV AB, Volvo Group AB, Swerea SWECAST and Jönköping University. All participating personell from these institutions/companies are greatly acknowledged.

\section{References}

[1] H. Fredriksson, U. Åkerlind, Mat. Proc. during casting. Vol. 210. Wiley Online Library. 2006.

[2] D. Stefanescu, Sci. and Eng. of casting solidification, third ed.: Springer. 2015

[3] G. Alonso, D. Stefanescu, R. Suarez, Int Foundry Res, 66 (2014) 2-12.

[4] J. Campbell, Chapter 7: Porosity, in Complete Casting Handbook, Butterworth-Heinemann: Oxford. 2011, 391-464.

[5] H. Fredriksson, J. Stjerndahl, and J. Tinoco, Mat. Sci. and Eng. A-Struct, 413 (2005) 363-372.

[6] A. Diószegi, L. Elmquist, and S. Adolfsson, AFS Trans., 105 (2008) 691-703.

[7] L. Elmquist and A. Diószegi, Mat. Sci. Forum, 649 (2010) 443-448.

[8] L. Elmquist, K. Soivio, and A. Diószegi, Mat. Sci. Forum. 2014. Trans Tech Publ.

[9] J. Powell, The British Foundryman, 77 (1984) 472-483.

[10] R. Lora, A. Diószegi, and L. Elmquist, Key Eng. Mat., 457 (2011) 108-113.

[11] J. Hernando, B. Domeij, D. González, J. M. Amieva, A. Diószegi, Metall. Mater Trans. A 48 (2017) 5432-5441.

[12] J.M. Motz, Practical Metallography, 25 (1988) 285-293.

[13] M. Gungor, Metall. Trans. A, 20 (1989) 2529-2533.

[14] A. Kagawa and T. Okamoto, J. of Mat. Sci., 19 (1984) 2307-2318.

[15] A. Diószegi and J. Hattel, Int. J. of Cast Met. Res., 17 (2013) 311-318.

[16] J.O. Andersson, et al., Calphad, 26 (2002) 273-312.

[17] S., Subramanian. Physical Metall. of Cast Iron IV, (1989) 73-80.

[18] E.N. Pan. K. Ogi, and C.R. Loper Jr., AFS Trans., 90 (1982) 509-527.

[19] B. Domeij, A. Diószegi, (2017). Solidification Processing 2017 (SP17), Old Windsor, London, UK, BCAST, Brunel University London.

[20] F. Mampaey. and Z.A. Xu, Cast Met., 7 (1994) 165-174. 